\title{
"-
}

\section{MINERÍA DE PROCESOS: DESARROLLO, APLICACIONES Y FACTORES CRÍTICOS*}

\author{
Hugo Santiago Aguirre Mayorga \\ Nicolás Rincón García ****
}

doi:10.11144/Javeriana.cao28-50.mpda. Este artículo es producto de la investigación “Desarrollo de una metodología para la aplicación de minería de procesos" financiado por la Pontificia Universidad Javeriana, desde el 1 de julio de 2013 hasta el 1 de diciembre de 2014. El artículo se recibió el 03/02/2015 y se aprobó el 30/05/2015. Sugerencia de citación: Aguirre M., H. S. y Rincón G., N. (2015). Minería de procesos: desarrollo, aplicaciones y factores críticos. Cuadernos de Administración, 28 (50), 137-157. http:// dx.doi. org/10.11144/Javeriana.cao28-50.mpda

** Ph. D. en Ingeniería de la Pontificia Universidad Javeriana, Bogotá, Colombia, 2015. Profesor Asociado de Ingeniería Industrial de la Pontificia Universidad Javeriana, Bogotá, Colombia.

Correo electrónico: saguirre@javeriana.edu.co

*** Estudiante de doctorado de la Universidad de Southampton, Inglaterra. Profesor Asistente de Ingeniería Industrial de la Pontificia Universidad Javeriana, Bogotá, Colombia.

Correo electrónico: nicolas.rincon@javeriana.edu.co 
Minería de procesos: desarrollo, aplicaciones y factores críticos

\section{RESUMEN}

La minería de procesos es una disciplina que tiene como objetivo descubrir, monitorear y mejorar procesos de negocio a través del análisis del registro de los eventos del proceso que se encuentran almacenados en los sistemas de información. En este artículo se presenta el desarrollo de la minería de procesos y se analizan tres casos de estudio de aplicación de esta disciplina para determinar los factores críticos que se deben considerar para su correcto uso. A partir de lo anterior se discute sobre los desafíos futuros que puede presentar la minería de procesos en su aplicación en las organizaciones y las investigaciones que se requieren para el avance de esta disciplina, que ha sido catalogada como la más promisoria en Business Process Management.

Palabras clave: minería de procesos, factores críticos, análisis de procesos.

Clasificación JEL: C18, M11, L80
Process mining: development, applications and critical factors

\section{Mineração de processos: desenvolvimento, aplicações e fatores críticos}

\begin{abstract}
Process mining is the discipline that aims at discovering, monitoring and improving business processes by analyzing event logs recorded by an information system. In this paper, the evolution of the concepts involved in process mining is presented along with the analysis of three case studies in order to understand the critical success factors in its implementation. Starting from this point, the future challenges that might be present in its application on organizations are discussed along with the required research to provide solutions to the industry in a discipline that has been considered as the most promising in the field of Business Process Management.
\end{abstract}

Keywords: Process mining, critical success factors, process analysis.

JEL Classification: $\mathrm{C}_{18}, \mathrm{M}_{11}$, L80

\section{Resumo}

A mineração de processos é uma disciplina que tem como objetivo descobrir, monitorar e melhorar processos de negócios por meio da análise do registro dos eventos do processo que se encontram armazenados nos sistemas de informação. Neste artigo, apresenta-se o desenvolvimento da mineração de processos e analisam-se três casos de estudo de aplicação dessa disciplina para determinar os fatores críticos que devem ser considerados para seu uso adequado. A partir disso, discute-se sobre os desafios futuros que a mineração de processos pode apresentar em sua aplicação nas grandes organizações e as pesquisas que se requerem para o avanço dessa disciplina, que tem sido catalogada como a mais promissora em Business Process Management.

Palavras-chave: mineração de processos, fatores críticos, análise de processos. Classificação JEL: C18, M11, L80 


\section{Introducción}

Actualmente los procesos de negocio de las organizaciones están soportados por sistemas de información que registran datos valiosos con respecto a ejecutores, actividades, eventos, tiempos y variables asociadas a la ejecución de los procesos. Esta información puede ser aprovechada con técnicas de la minería de datos, más específicamente, de la minería de procesos para descubrir la realidad de cómo se están ejecutando los procesos y de esta forma tomar decisiones para mejorarlos (Van der Aalst, 2011).

Los algoritmos y herramientas de la minería de procesos se empezaron a desarrollar por parte de grupos de investigación en la academia (Van der Aalst y Weiters, 2004) y paulatinamente se han transferido a la industria a través de casos de estudio y proyectos de minería de procesos. En la última década los esfuerzos de los investigadores han estado encaminados principalmente a desarrollar y probar algoritmos para superar las limitaciones y problemas encontrados en las técnicas por lo que algunos autores enfatizan en la necesidad de tener más estudios prácticos para probar los beneficios de la minería de procesos en casos reales (Weerdt et al., 2013; Van der Aalst et al., 2012; Bozkaya et al., 2009).

Por otro lado, en el primer Manifiesto de la Minería de Procesos (Van der Aalst et al., 2012) publicado por el grupo de trabajo en minería de procesos del IEEE (task force on process mining), los autores identificaron once retos para el avance de la disciplina, y uno de ellos consiste en incrementar el entendimiento de la minería de procesos y su utilización por parte de no expertos.

Atendiendo a lo anterior, en los últimos años se han desarrollado metodologías que definen una serie de etapas, pasos y lineamientos para la aplicación de la minería de procesos en una organización (Van Eck et al., 2015; Aguirre, 2015). El objetivo de estas metodologías es facilitar la aplicación de estas técnicas para el mayor desarrollo de la disciplina en las organizaciones. A pesar de esto, los resultados y retos en cada caso de aplicación han sido diversos por lo que en este artículo se determinan los factores críticos que se deben tener en cuenta para que los proyectos de minería de procesos generen los resultados esperados.

Para esto se parte de analizar el concepto, origen y desarrollo de la minería de procesos, lo que se presenta en la primera sección de este artículo. En la segunda se analizan tres casos de aplicación desarrollados con una metodología diseñada para el efecto (Agui- 
rre, 2015) y se comparan con otros casos de aplicación. A partir de esto se determinan los factores críticos en la aplicación de la minería de procesos lo que se presenta en la tercera sección. Para terminar, se discuten los desafíos y retos investigativos en la aplicación de la disciplina.

\section{Minería de procesos}

\subsection{Concepto de minería de procesos}

La minería de procesos es una disciplina que tiene como objetivo descubrir, monitorear y mejorar procesos a través de la extracción de conocimiento del registro de eventos de los sistemas de información (Van der Aalst, 2011).

Este "registro de eventos" (event log) corresponde al histórico de ejecución de los procesos de negocio, donde se encuentran las instancias o casos del proceso (ej. Solicitud de compra 1451), las actividades del proceso (ej. Envío de orden de compra al proveedor), las personas que ejecutan cada actividad (ej. Carlos Pérez - Analista de compras), el inicio y fin de cada actividad (ej. Inicio: $21 / 11 / 2011 ; 14: 51: 00$, Fin: 21/11/2011; 17:01:02) y otros datos asociados al caso (ej. Producto a comprar, proveedor, departamento solicitante).

Los registros de eventos se encuentran disponibles en los sistemas PAIS (Process Aware Information Systems), como son los sistemas de workflow, BPMS, ERP, CRM, entre otros (Dumas et al., 2005). Esta información podría ser analizada con técnicas de minería de procesos para hacer explícita información que genere conocimiento con el fin de que las organizaciones comprendan y mejoren sus procesos.

Con la minería de procesos las organizaciones pueden, entre otras cosas:

- Descubrir el modelo de ejecución real del proceso. A través de algoritmos de minería de procesos aplicados al análisis de los registros de eventos, se puede descubriry llegar al modelo real de un proceso. Este modelo puede ser expresado en términos de una red de Petri o en la notación BPMN. El argumento de la minería de procesos es que este modelo describe la situación real y no se basa en la documentación de cómo se debería ejecutar el proceso o en la percepción de las personas (Rozinat, 2008). 
- Determinar si el proceso cumple con la reglamentación y procedimientos documentados. Al tener el modelo real de un proceso se puede comparar con los procedimientos documentados para determinar si se está cumpliendo con los estándares, protocolos, reglamentación y políticas de ejecución de un proceso. Es posible detectar y mitigar posibles fuentes de no conformidad y fraudes (Jans et al., 2011).

- Analizar la interacción del personal que ejecuta el proceso. A través de la aplicación de técnicas de minería de datos se puede construir la red social del proceso (social network) para analizar la interacción entre los individuos y descubrir bucles (loops) que pueden demorar la ejecución de un proceso (Van der Aalst et al., 2007; Van der Aalst y Song, 2008).

- Descubrir cuellos de botella. A través de la animación del flujo de los casos reales del proceso se pueden determinar los cuellos de botella para actuar sobre estos y mejorar el nivel de desempeño del proceso (Aguirre et al., 2013).

- Monitorear la productividad del personal. A través del análisis de los registros de ejecución del proceso se pueden descubrir los datos de productividad real de las personas que ejecutan el proceso, así como los tiempos de ciclo por actividad (Aguirre et al., 2013). Con esto ya no será necesario hacer estudios de tiempos y movimientos de forma "manual".

- Predecir el tiempo de ciclo de un caso. A través de la aplicación de técnicas de la minería de datos como son los árboles de decisión se puede predecir el tiempo de ejecución restante de un proceso. Por ejemplo, se podría dar una respuesta satisfactoria al cliente que llame a un call center para saber cuándo va a estar procesada su solicitud de crédito en un banco (Van der Aalst et al., 2011).

- Determinar la relación entre las variables de un caso. A través de la aplicación de técnicas de clasificación se puede determinar cómo diferentes variables asociadas a un caso (por ejemplo: tipo de producto a comprar, comprador, proveedor, departamento solicitante), pueden influir en los tiempos de ciclo de un proceso (Aguirre et al., 2013).

El objetivo final de la minería de procesos es el mejoramiento de los procesos a través de la aplicación de herramientas de análisis de datos. En la Figura 1 se presenta esquematizada la minería de procesos en sus tres componentes (Van der Aalst, 2011); Descubrir (Discovery), Cumplimiento (Conformance) y Mejora del proceso (Enhacement). 


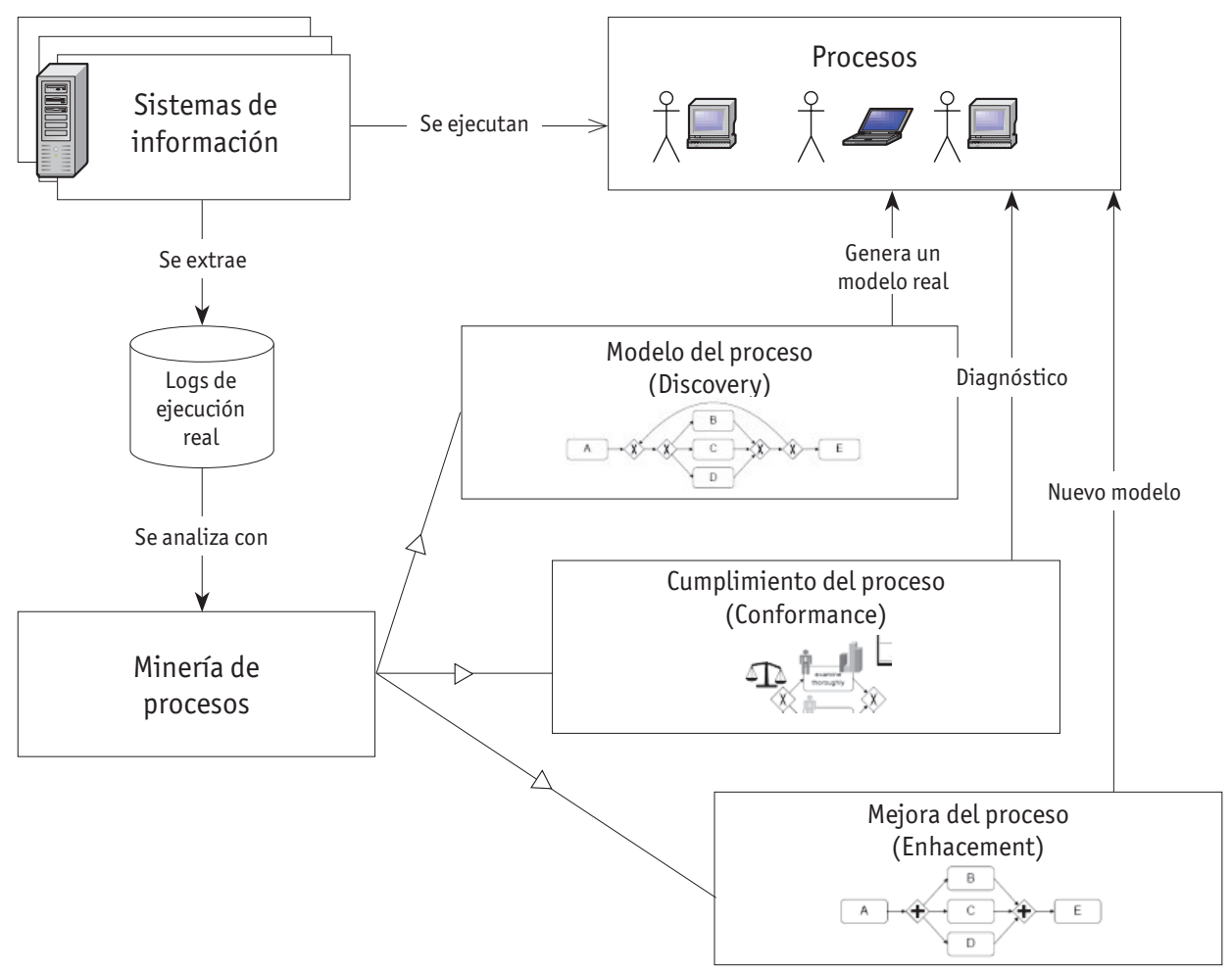

Figura 1. Esquema de la minería de procesos

Fuente: elaboración propia.

La minería de procesos es una disciplina reciente que se encuentra entre la minería de datos y el modelamiento y análisis de procesos (Van der Aalst, 2011).

\subsection{Desarrollo de la minería de procesos}

La aplicación de la minería de datos para el análisis de procesos se remonta a 1998 donde se dan a conocer los primeros trabajos de aplicación de minería de datos para el análisis de flujos de trabajo (Agrawal et al., 1998). De otro lado, Cook y Wolf (1998) investigaron la minería de procesos aplicada en el contexto de la ingeniería de software.

A partir de esto se empezaron a desarrollar trabajos orientados a perfeccionar los algoritmos para manejar aspectos de los procesos como son la concurrencia, actividades duplicadas, ruido, entre otros. Estos temas y otros retos investigativos fueron expuestos 
en el artículo Process mining: A research agenda, de los profesores Van der Aalst y Weijters (2004). Este artículo se puede considerar como una de las publicaciones referente en el tema y fue publicado como una edición especial en la revista Computers in Industry, junto con otros artículos de investigación en la temática (Herbst y Karagiannis, 2004; Shimm, 2004; Pinter y Golani, 2004; Grigori et al., 2004; Hwang et al., 2004).

En los años posteriores se publicaron artículos con avances relevantes en la temática y con aplicaciones específicas de la minería de procesos (Van der Aalst et al., 2007; Mans et al., 2008; Maruster y Van Best, 2009; Rozinat et al., 2010; Jans et al., 2011; Van der Aalst et al., 2011; Rebuge y Ferreira, 2012; Aguirre et al., 2013, Weerdt et al., 2013) para descubrir y modelar procesos, predecir tiempos, así como también para el análisis de la perspectiva organizacional. Para seguir avanzando en el tema, el Comité Técnico de Minería de Datos (DMTC) del Instituto de Ingenieros Eléctricos y Electrónicos (IEEE) estableció en el 2009 el IEEE task force on process mining con el objetivo de "promover la investigación, desarrollo, educación y entendimiento de la minería de procesos" (Van der Aalst et al., 2012). En este equipo participan investigadores de universidades, representantes de proveedores de software, empresas y firmas de consultoría. Como primer resultado, este equipo publicó el primer Process mining manifesto (Van der Aalst et al., 2012), donde se expone el estado del arte, los principios guías y retos de la disciplina.

Por otro lado, Van der Aalst publica el primer libro en el tema titulado Process Mining: Discovery, Conformance and Enhancement of Business Process de la editorial Springer (Van der Aalst, 2011). En el 2014 y bajo el liderazgo del profesor Van der Aalst se ofrece el primer curso el línea en modalidad M00C de minería de procesos. Esto sin lugar a dudas ha contribuido con la expansión de la disciplina en la academia y organizaciones a través de desarrollo de proyectos y casos de estudio que han presentado diferentes retos que deben ser analizados.

\section{Análisis de los casos de estudio}

Para asegurar la representatividad de los casos de estudio se analizaron las variables que se deben considerar y su impacto en cómo se debe ejecutar un proyecto de minería de procesos en una organización. En la Tabla 1 se presenta el análisis de estas variables.

El sector de la empresa y el tipo de proceso a analizar tiene un bajo impacto en el cómo ejecutar un proyecto de minería de procesos dado que se deben seguir los mismos pasos independiente si la empresa es de servicios, manufactura o si pertenece a cierto sector 
industrial como salud, educación, consumo masivo, etc. En los casos prácticos analizados no se propone alguna metodología o técnica para cada sector o tipo de proceso. Para ciertos casos se podría recomendar alguna técnica para un proceso en un sector específico, como por ejemplo el uso de análisis de conglomerados para dividir los registros de eventos y agruparlos por procedimientos quirúrgicos en un proceso del sector salud (Rebuge y Ferreira, 2012).

\section{Tabla 1}

Impacto de las variables para el diseño de los casos de estudio

\begin{tabular}{lll}
\hline \multicolumn{1}{c}{ Variable } & \multicolumn{1}{c}{ Característica } & Impacto en minería de procesos \\
\hline Sector de la empresa & $\begin{array}{l}\text { Empresa del sector salud, educación, energía, } \\
\text { consumo masivo, servicios públicos, etc. }\end{array}$ & Bajo \\
Sistema fuente de los datos & $\begin{array}{l}\text { Sistema de información que soporta el proceso: } \\
\text { ERP, BPMS, otros. }\end{array}$ & Medio \\
Tipo de proceso & $\begin{array}{l}\text { Tipo de proceso a analizar: operativos, de cara al } \\
\text { cliente, de soporte. }\end{array}$ & Bajo \\
Tipo de proyecto de minería & $\begin{array}{l}\text { Tipo de proyecto: basado en datos, basado en } \\
\text { de procesos }\end{array}$ & Alto \\
\hline
\end{tabular}

Fuente: elaboración propia.

El sistema fuente de los datos tiene un impacto medio dado que dependiendo de esto se deben seguir ciertos pasos específicos para la localización y extracción de datos. Por ejemplo, en el sistema ERP de SAP se deben localizar los campos relevantes asociados a las transacciones para proceder a la extracción de los datos de las tablas y estructuras de datos. En este tipo de sistemas generalmente no se dispone de la fecha de inicio de cada actividad sino únicamente la fecha en que se registra la transacción que corresponde a la fecha fin de la actividad (Aguirre, 2015). Esto determina las herramientas que se pueden usar en la minería de procesos, como por ejemplo, en el análisis de desempeño sólo se puede estudiar el tiempo entre actividades y no el tiempo de duración de cada actividad.

\subsection{Tipos de proyectos de minería de procesos}

Según Van der Aalst (2011), existen tres tipos de proyectos de minería de procesos:

- Basados en preguntas: son proyectos donde se tienen hipótesis o preguntas que se quieren responder, comprobar o rechazar con base en la minería de procesos.

- Basados en objetivos: son proyectos donde se establecen como objetivos el mejoramiento de los indicadores clave de desempeño del proceso. 
- Basados en datos: son proyectos donde no hay unos objetivos establecidos, por lo que son de carácter exploratorio donde se pretende descubrir aspectos importantes con respecto a la ejecución real de los procesos.

Los proyectos basados en datos no tienen una fase de definición del proyecto, dado que por su naturaleza exploratoria se parte de unos datos y se debe realizar un análisis de minería de procesos para encontrar patrones y hallazgos que se usan para el diagnóstico del proceso. Por otro lado, los proyectos basados en objetivos hacen énfasis en mejorar el desempeño en los indicadores de gestión por lo que se debe hacer el análisis de brechas en los indicadores, a diferencia de los proyectos basados en preguntas para los cuales no aplica este paso.

Considerando lo anterior se diseñaron los casos de estudio para abarcar los tres tipos de proyecto de minería de procesos. En estos casos de estudio se aplicó la metodología que se explica en el siguiente punto.

\subsection{Descripción de la metodología}

La metodología aplicada para el desarrollo de cada uno de los casos de estudio es la propuesta por Aguirre (2015) en su tesis doctoral. En la Figura 2 se representa la metodología.

La metodología diseñada consta de cuatro etapas como se explica a continuación:

- Definición del proyecto. El objetivo de esta etapa es entender el proceso y sus principales problemas para, de esta forma, determinar los objetivos de mejora o las preguntas a responder con la aplicación de la minería de procesos.

- Preparación de los datos. El objetivo de esta etapa es localizar los datos requeridos para el análisis, extraerlos del sistema de información y asegurar su calidad para el posterior análisis con las técnicas de minería de procesos.

- Análisis del proceso. En esta etapa se aplican las técnicas de minería de procesos para descubrir el modelo real de ejecución del proceso, analizar su desempeño, las interacciones entre las personas involucradas en el proceso y verificar si se están cumpliendo los procedimientos y las reglas de negocio establecidas.

- Rediseño del proceso. El objetivo de esta etapa es determinar alternativas de mejora basado en los hallazgos de la etapa anterior, evaluar su factibilidad e implantar las mejoras. 


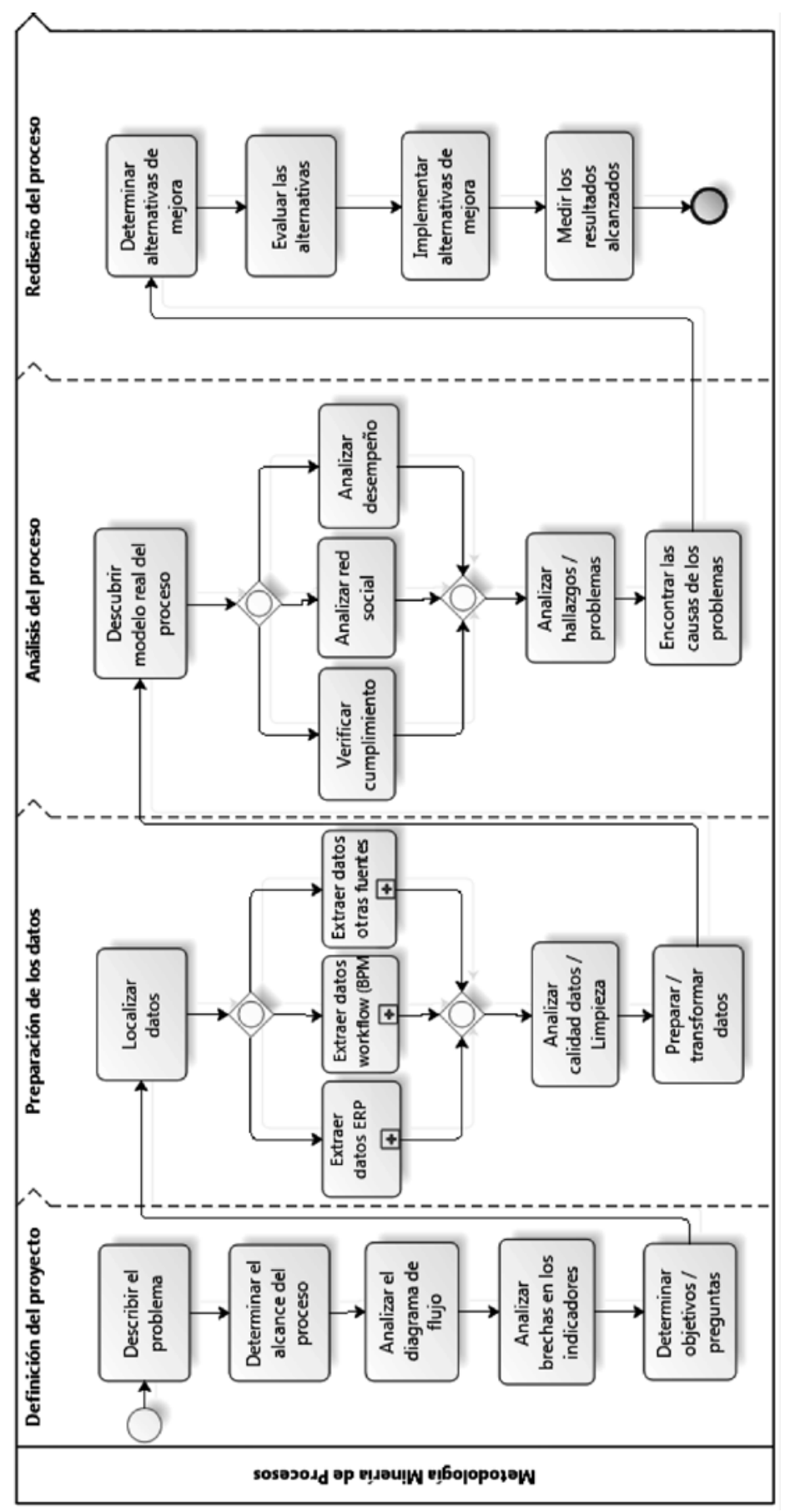

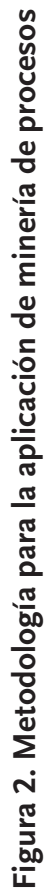


Cada etapa tiene unos pasos específicos que fueron aplicados en los casos de estudio que se analizan en detalle en el siguiente punto.

\subsection{Casos de estudio: aplicación y retos}

El caso de estudio 1 corresponde a la aplicación de la metodología de minería de procesos en el proceso de ventas y distribución en una empresa comercializadora. La organización tuvo un proceso de fusión con la operación de otra compañía del mismo sector y el principal interés en la aplicación de la minería de procesos fue la de conocer el nivel de desempeño del proceso fusionado, por lo que corresponde a un proyecto basado en preguntas. El proceso está soportado por el sistema ERP de SAP.

A manera de ejemplo, en la Figura 3 se presenta el descubrimiento del proceso del caso de estudio 1, donde se encuentra un extracto de la modelación del proceso teórico en la notación BPMN, que corresponde a la secuencia de actividades y decisiones mediante las cuales se debe ejecutar el proceso analizado. En la parte de abajo de la figura se encuentra el modelo real de ejecución del proceso, resultado de la aplicación de un algoritmo de la minería de procesos, representado en un diagrama de secuencias, donde se pueden visualizar las actividades representadas por rectángulos, en los que se identifica el nombre de la actividad y la cantidad de casos procesados. En las flechas de flujo se encuentran el número de casos que fluye de una actividad a otra.

El análisis de estos diagramas permitió verificar las diferencias entre el proceso teórico y el proceso real, donde se puede comprobar que en algunos casos no se estaba cumpliendo la regla de negocio de que los pedidos deben liberarse por el área de cartera (actividad liberación orden), antes de la preparación del despacho (actividad entrega de salida).

El caso de estudio 2 corresponde a la aplicación de la metodología y las técnicas de minería de procesos en la compra de bienes de una institución universitaria. A pesar de que este proceso está soportado y estandarizado en el sistema ERP Peoplesoft, presenta alta complejidad debido al volumen de compras que se debe realizar y a la variedad de bienes y productos que se requieren para el funcionamiento de las Facultades y Departamentos de la universidad. En este caso se planteó un objetivo principal para el proyecto: disminuir los tiempos de ciclo del proceso. 

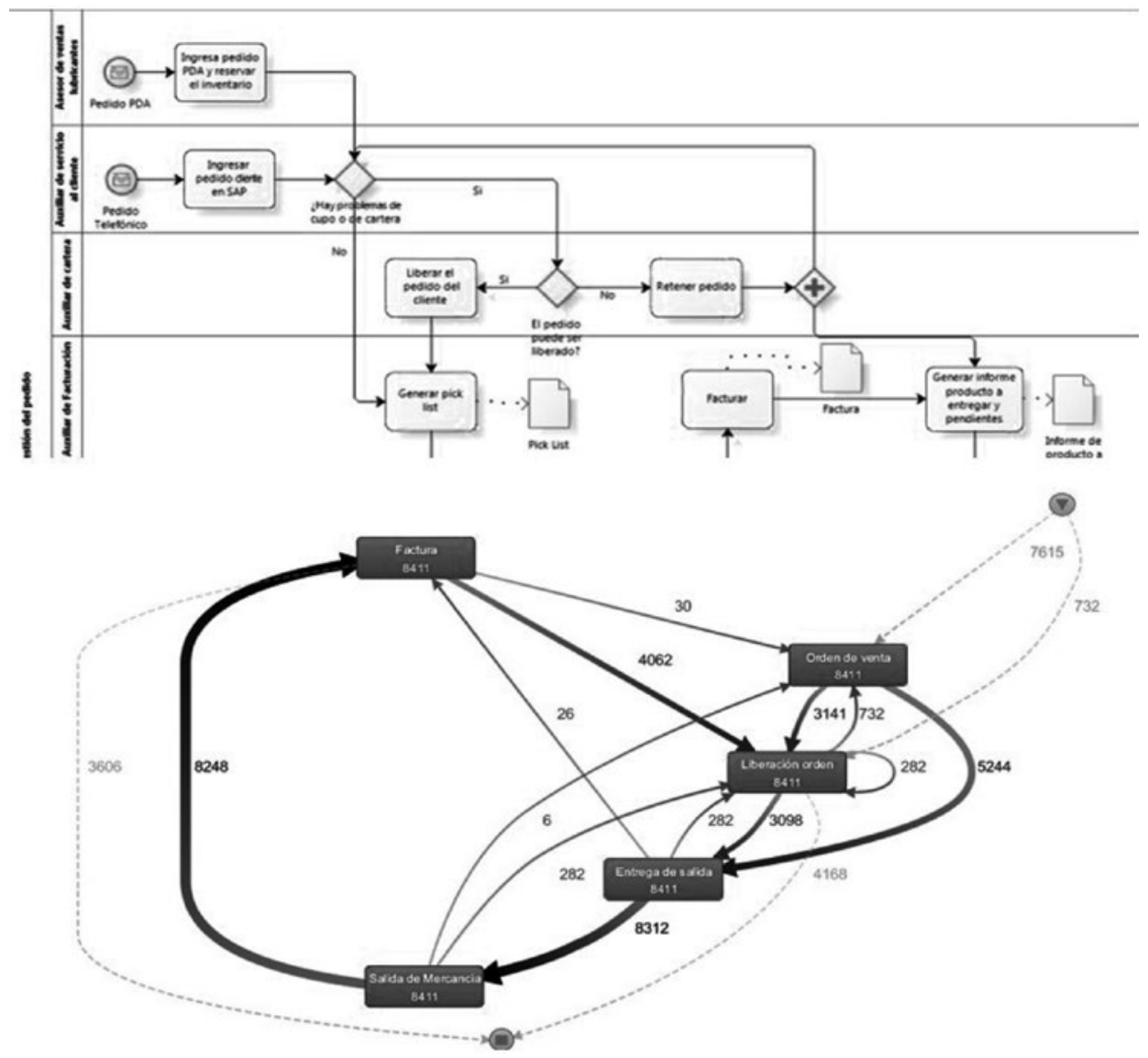

Figura 3. Descubrimiento del proceso en el caso de estudio 1

Fuente: elaboración propia.

El caso de estudio 3 corresponde a un proyecto basado en datos dado que la principal motivación es encontrar información valiosa sobre el desempeño de un proceso basado en el análisis del registro de eventos de un sistema BPMS. Este caso surge por la gestión realizada con un proveedor de software BPMS para analizar cómo se puede aplicar la minería de procesos con los datos provenientes de su sistema de información. Para este caso se tuvo un alto nivel de confidencialidad de la información por lo que no se pueden presentar diagramas ni datos específicos del análisis. De acuerdo con la información entregada, el proceso analizado corresponde a una empresa de servicios. En la Figura $4 \mathrm{se}$ representan las principales características de cada uno de los pasos y las técnicas usadas. 


$\begin{array}{llll} & \end{array}$

Figura 4. Descripción de los casos de estudio

Fuente: elaboración propia.

En los casos de estudio analizados se aplicaron diferentes técnicas de minería de procesos y de minería de datos como las siguientes:

- Algoritmo fuzzy. Permitió descubrir el modelo real de ejecución del proceso.

- Árboles de decisión. Se usó para encontrar las variables que tienen mayor incidencia en la probabilidad de que un caso termine antes de cierto tiempo.

- Análisis de la red social. Estos algoritmos permitieron descubrir los cargos que tienen mayor centralidad en el proceso.

- Análisis estadístico. Permitió analizar el nivel de variabilidad de los procesos y encontrar diferencias en la productividad del personal.

Estas técnicas fueron aplicadas con el soporte de las siguientes herramientas informáticas: Disco, SPSS y Microsoft Excel. A partir de los hallazgos se determinaron las causas para plantear alternativas de mejora. 


\subsubsection{Retos CASOS De ESTUdio 1}

En este caso de estudio la etapa que requirió mayor esfuerzo y dedicación en términos de tiempo fue la de preparación de los datos, esto debido a la complejidad y estructura del sistema SAP ERP. El paso de transformar los datos requirió el desarrollo de algoritmos para adecuar el reporte de SAP ERP a la estructura de datos que requiere el software de minería de procesos, lo que consumió buena cantidad de tiempo. A continuación se explican los retos y las lecciones aprendidas de este caso:

- Para la localización de los datos en el sistema ERP de SAP es necesario previamente modelar el proceso de negocio en una notación que permita identificar la secuencia de actividades del proceso y los documentos que se generan en cada paso para identificar las transacciones del sistema en donde se localizan los datos.

- En la etapa de localización de los datos de un sistema ERP es de vital importancia involucrar al personal de la organización que maneja el sistema de información y que conoce el proceso de negocio para asegurar que se están localizando los datos correctos, dado que el sistema tiene muchos campos similares $(v$. gr. fecha de transacción, fecha de registro, fecha de la orden), y cada uno tiene su significado.

- La localización y extracción de datos del sistema ERP de SAP tiene un alto nivel de complejidad y se requiere el conocimiento de las tablas y estructuras de datos para poder desarrollar los reportes o consultas requeridos para la extracción de los datos.

\subsubsection{RETOS CASO DE ESTUdio 2}

Este caso de estudio permitió la aplicación de todos los pasos de las etapas definición del proyecto, preparación de los datos y análisis del proceso. La etapa de preparación de los datos se facilitó al disponer de un reporte prediseñado en el sistema ERP Peoplesoft con toda la información requerida. Sin embargo, se tuvo problemas con la calidad de los datos por lo que se realizó un exhaustivo análisis para limpiar los casos con datos perdidos, inconsistentes o atípicos. Enseguida se presentan los retos y las lecciones aprendidas de este caso:

- Uno de los aspectos críticos para el análisis con minería de procesos es la calidad de los datos. Se debe hacer un análisis exhaustivo para asegurar la confiabilidad, completitud y validez de los datos. El trabajo en conjunto con los ejecutores del 
proceso es fundamental para asegurar que los datos correspondan a la realidad de la ejecución del proceso.

- La minería de procesos revela las diferencias en la productividad del personal o identifica los cargos que por alguna causa demoran la ejecución de los procesos. Esta información debe manejarse con confidencialidad debido a la privacidad y a las leyes laborales por lo que se deben determinar las posibles razones de las diferencias en productividad con el personal involucrado, antes de presentarlo a la gerencia.

\subsubsection{Retos caso de estudio 3}

En este caso de estudio no se realizó la etapa de definición debido a la naturaleza del proyecto. Este caso inició directamente con la etapa de preparación de los datos que no requirió mayor tiempo o esfuerzo, dado que la estructura de la base de datos del sistema BPMS facilitó el desarrollo de la consulta para extraer la información.

La etapa que requirió mayor tiempo y esfuerzo fue la del análisis del proceso, dado que no se contó con una línea base de comparación, como por ejemplo la modelación o diagrama de flujo del deber ser del proceso. En este proyecto se requirió combinar técnicas tradicionales de minería de datos (árboles de decisión) con técnicas específicas de minería de procesos (algoritmo fuzzy, análisis de la red social, análisis de tiempos y productividad). Los retos y las lecciones aprendidas de este caso son:

- Los proyectos basados en datos son de tipo exploratorio donde no se tiene una base cuantitativa para evaluar el éxito del proyecto o el cumplimiento de objetivos. Este tipo de proyectos no parten de unos objetivos o preguntas a resolver por lo que no se puede medir el impacto del proyecto más allá de los hallazgos encontrados de la etapa del análisis del proceso.

- En los proyectos basados en datos no es relevante la etapa de definición del proyecto dado que no están apalancados por objetivos de mejora sino por transformar los datos en información y hallazgos. Este tipo de proyectos inician con la localización y extracción de datos.

- La estructura a nivel de base de datos del sistema BPMS de Bizagi facilita la identificación de las tablas para la extracción de datos.

- Los sistemas BPMS registran de forma sistemática y en una estructura lógica toda la información requerida para el análisis de minería de procesos. 


\section{Factores críticos para la aplicación de la minería de procesos}

Los casos de aplicación de la minería de procesos permitieron determinar los factores críticos que se deben tener en cuenta para su aplicación en un contexto organizacional. A continuación se presentan estos factores:

- Los proyectos de minería de procesos deben tener un impacto sobre los indicadores estratégicos del negocio. Para asegurar la adecuada alineación del proyecto de minería de procesos con la estrategia de la organización se debe realizar el análisis de brechas para determinar el nivel de desempeño esperado en los indicadores de gestión claves del proceso. Esto es determinante para el establecimiento de los objetivos y preguntas a resolver con el proyecto de minería de procesos.

- El entendimiento del flujo del proceso a través de su modelación es un aspecto fundamental para la localización de los datos de los sistemas ERP que se requieren para el análisis con las técnicas de minería de procesos. La modelación del proceso idealizado provee las actividades, eventos y decisiones del proceso, así como el flujo de datos y documentos. El flujo de datos y documentos permite establecer las transacciones del sistema ERP donde se localizan los datos necesarios para la construcción del registro de eventos del proceso.

- Los objetivos y preguntas del proyecto de minería de procesos son determinantes para la localización y selección de los datos a extraer en la etapa de preparación de los datos. De igual forma, el análisis del proceso debe estar orientado a responder las preguntas y a encontrar hallazgos que contribuyan con el mejoramiento de los indicadores de gestión del proceso asociados a los objetivos del proyecto.

- En la localización de los datos en los sistemas ERP es necesario y determinante involucrar a las personas que ejecutan el proceso así como al personal de tecnología informática de la organización, para encontrar las transacciones, tablas, estructuras de datos y campos donde se encuentra la información requerida para el análisis con minería de procesos. La configuración del sistema ERP varía en cada organización por lo que es difícil desarrollar una herramienta y/o aplicar un procedimiento estándar.

- La calidad de los resultados de la minería de procesos está directamente relacionada con la calidad de los datos con los cuales se realiza el análisis. De acuerdo con esto, uno de los pasos críticos es analizar la calidad y limpiar los datos, para lo cual son de gran utilidad las herramientas estadísticas para la identificación de los casos con datos atípicos e involucrar al personal de la organización para decidir sobre su inclusión o eliminación. 
- La selección de las técnicas y algoritmos para el análisis con minería de procesos debe estar asociada a los objetivos del proyecto y a las preguntas que se quiera resolver. Los hallazgos del análisis del proceso se deben priorizar para profundizar en los problemas que tengan relación con estos objetivos.

- Los proyectos basados en datos o exploratorios tienen algunas desventajas, dado que no se pueden determinar los criterios de éxito del proyecto. En estos no se dispone de unos objetivos o preguntas para poder evaluar los resultados obtenidos. Por otro lado, se presentan dificultades al escoger las herramientas que se deben usar en el análisis del proceso, dado que no está claro qué se quiere buscar. En este tipo de proyectos es recomendable hacer el esfuerzo para entender los principales problemas del proceso y analizar las brechas de desempeño de forma que se puedan transformar en proyectos basados en objetivos o preguntas.

- Es fundamental involucrar al personal de la organización, tanto a nivel gerencial como operativo, para la determinación y evaluación de las alternativas de mejora. Involucrar al personal operativo en esta etapa facilita la implantación de las alternativas al disminuir la resistencia al cambio.

\section{Desafíos y retos investigativos}

En cada caso de estudio se tuvo un sistema de información fuente de donde se extrajeron los datos para el análisis. Estos corresponden a los sistemas ERP (SAP y Peoplesoft) y el sistema BPMS de Bizagi, por lo que los lineamientos y recomendaciones para la extracción de datos se basan en lo encontrado en estos sistemas de información y en la revisión del estado del arte.

Una de las conclusiones de esta investigación es que cada sistema de información tiene una estructura diferente y no hay uniformidad en la manera que registran los eventos del proceso. De acuerdo con esto, esta investigación estuvo orientada a tres casos de estudio con los sistemas de información anteriormente mencionados.

Uno de los desafíos más importantes de la minería de procesos es contar con mejores herramientas para la localización y extracción de datos (Van der Aalst, 2015), por lo que un trabajo futuro que tendría gran contribución para avanzar en esta temática sería el de comparar la forma y estructura como registran los eventos del proceso diferentes sistemas de información, para desarrollar metodologías y herramientas específicas que permitan localizar y extraer los datos en menor tiempo. 
Por último se debe considerar que la minería de procesos es una disciplina relativamente nueva en cuanto a su aplicación en contextos empresariales. Se considera que para el avance de la disciplina se requiere mayor trabajo en conjunto entre la academia y las organizaciones para, basado en sus necesidades, mejorar las técnicas, los algoritmos, etc., lo que llevará a mejorar la funcionalidad de los aplicativos.

\section{Conclusiones}

La minería de procesos es una disciplina que provee una serie de técnicas, algoritmos y herramientas que permiten a las organizaciones analizar los procesos de negocio basados en los datos de ejecución real almacenados en los sistemas de información.

Las organizaciones en las que se han aplicado estas técnicas han descubierto el modelo de ejecución real del proceso, han determinado el nivel de cumplimiento de las normas y reglas de negocio, encontraron los cuellos de botella del proceso, analizaron la interacción de los ejecutores del proceso y determinaron las variables que influyen en los tiempos de ciclo. Este análisis les sirvió para mejorar sus procesos de negocio.

Uno de los factores críticos que determinan la calidad de los resultados de la minería de procesos es la calidad de la información con la que hace el análisis. Por lo tanto, es muy importante depurar y limpiar los datos, para lo cual son de gran utilidad las herramientas estadísticas para la identificación de los casos con datos atípicos e involucrar al personal de la organización para decidir sobre su inclusión, imputación o eliminación.

El análisis del flujo del proceso a través de su modelación es un aspecto fundamental para el entendimiento del proceso y para determinar las transacciones de los sistemas de información de los cuales se va a extraer los datos de ejecución real del proceso. De igual forma el modelo teórico del proceso es la base de comparación para determinar si el modelo real del proceso cumple con lo establecido en los procedimientos.

Los objetivos y las preguntas del proyecto de minería de procesos son determinantes para la localización y selección de los datos a extraer en la etapa de preparación de los datos. De igual forma el análisis del proceso debe estar orientado a responder las preguntas planteadas y a encontrar hallazgos que contribuyan con el mejoramiento de los indicadores del proceso asociados a los objetivos del proyecto. 
El involucramiento del personal de la organización, tanto a nivel gerencial como operativo, es fundamental en todas las etapas de un proyecto de minería de procesos. Esto facilita determinar los objetivos del proyecto y localizar los datos, así como también facilita la implantación de las alternativas de mejora al disminuir la resistencia al cambio en el rediseño del proceso.

En suma, la minería de procesos ha venido madurando en sus técnicas y herramientas y como consecuencia ha tenido una creciente adopción por parte de las organizaciones y de los proveedores de tecnología. Los beneficios que han obtenido las empresas que han aplicado la minería de procesos convierten a esta disciplina en la de mayor potencial a futuro en el área del Business Process Management (BPM).

\section{Referencias}

Agrawal, R., Gunopulos, D., and Leymann, F. (1998). Mining process models from workflow logs. En: Sixth International Conference on Extending Database Technology (pp. 469-483). London: Springer.

Aguirre, S. (2015). Metodología para la aplicación de minería de procesos. Tesis doctoral. Bogotá: Pontificia Universidad Javeriana.

Aguirre, S., Parra, C., and Alvarado, J. (2013), Combination of process mining and simulation techniques for business process redesign: A methodology approach. Lecture notes in business information processing, 162, 24-43.

Bozkaya, M., Gabriels. J., and van der Werf, J. (2009). Process diagnostics: A method based on process mining. In: Proceedings International Conference on Information, Process and Knowledge Management 2009. Cancun: IEEE.

Cook, J., and Wolf, A. (1998). Discovering models of software processes from event-based data. ACM Transactions on Software Engineering and Methodology, 7 (3), 215-249.

Dumas, M., La Rosa, M., Mendling, J., and Reijers, H. (2013). Fundamentals of Business Process Management. Berlin: Springer.

Dumas, M., van der Aalst W.M.P., and Hofstede, A. (2005) Process-aware information systems: Bridging people and software through process technology. New Jersey: Willey.

Grigori, D., Casati, F., Castellanos, M., Dayal, U., Sayal, M., and Shan, M. (2004). Business process intelligence. Computers in industry, 53 (3), 321-343.

Herbst, J., and Karagiannis, D. (2004). Workflow mining with InWoLvE. Computers in Industry. 53 (3), 245-264.

Hwang, S., Wei, C., and Yang, W. (2004). Discovery of temporal patterns from process instances. Computers in Industry, 53 (3), 345-364. 
Jans, M., Van der WerfJ., Lybaert, N., and Vanhoof, K. (2011). A business process mining application for internal transaction fraud mitigation. Expert Systems with Applications, 38 (10), 13351-13359.

Maruster, L., and Van Beest, L. (2009). Redesigning business processes: A methodology based on simulation and process mining techniques. Knowledge and Information Systems, 21 (3), 267-297.

Mans, R., Schonenberg, M., Song, M., Van der Aalst, W.M.P., and Bakker, P. (2008). Application of process mining in healthcare. A case study in a Dutch Hospital. In: A. Fred, J. Filipe, and H. Gamboa (eds.) BIOSTEC 2008 (pp. 425-438). Madeira: IEEE.

Pinter, S., and Golani, M. (2004). Discovering workflow models from activities' lifespans. Computers in Industry, 53 (3), 283-296.

Rebuge, A., and Ferreira, D. (2012). Business process analysis in healthcare environments: A methodology based on process mining. Journal Information Systems, 37 (2), 99-116.

Rozinat, A. (2008). Discovering colored Petri nets from event logs. International Journal of Software Tools for Technology Transfer, 10 (1), 57-74.

Rozinat, A., Jong, I., and Gunther, C. (2010). Process mining applied to the test process of wafer steppers in ASML. IEEE Transactions on Systems Man and Cybernetics, 1-6.

Schimm, G. (2004). Mining exact models of concurrent workflows. Computers in Industry, 53 (3), 265-281.

Van der Aalst, W.M.P. et al. (2012). Process Mining Manifesto. In: F. Daniel, K. Barkaoui, and S. Dustdar (eds). Business Process Management Workshops (pp. 169-174). Berlin: Springer.

Van der Aalst, W.M.P., Reijersa, H., Weijtersa, A., Dongena, V., Alves de Medeirosa, A., Songa, M., and Verbeeka, H. (2007). Business process mining: An industrial application. Information Systems, 32 (5), 713-732.

Van der Aalst, W.M.P. (2015). Extracting event data from databases to unleash process mining. In: J. Broke and T. Schmiedel (eds.). BPM-Driving innovation in a digital world (pp. 105128). Heidelberg: Springer.

Van der Aalst, W.M.P, Schonenberg, M., and Song, M. (2011). Time prediction based on process mining. Information Systems, 36 (2), 450-475.

Van der Aalst, W.M.P. (2011). Process mining: Discovery, conformance and enhancement of business process. Berlin: Springer.

Van der Aalst, W.M.P., and Weijters, A.J.M.M. (2004). Process mining: A research agenda. Computers in Industry, 53 (3), 231-244.

Van der Aalst, W.M.P., Weijters, A., and Maruster, L. (2004). Workflow mining: Discovering process models from event logs. IEEE Transactions on Knowledge and and Data Engineering, $16(9), 1128-1142$. 
Van der Aalst, W.M.P., and Song, M. (2008). Towards comprehensive support for organizational mining. Decision Support Systems, 46 (1), 300-317.

Van Eck, M., Xixi, L, Leemans, S., and van der Aalst, W.M.P. (2015). PM2: A process mining project methodology. Lecture Notes in Computer Science, 9097, 297-313.

Weerdt, J., Schuppa, A., Vanderloock, A., and Baesensa, B. (2013). Process mining for the multi-faceted analysis of business processes. A case study in a financial services organization. Computers in Industry, 64 (1), 57-67. 
